\title{
Transition Radiation from the Neutrino-Photon Interaction in Matter
}

\author{
Juan Carlos D'Olivo* and José Antonio Loza ${ }^{\dagger}$ \\ Instituto de Ciencias Nucleares,Universidad Nacional Autónoma de México, \\ Apartado Postal 70-543, 04510 México, D.F., Mexico
}

\begin{abstract}
We show that, because of their effective electromagnetic interaction in matter, transition radiation is emitted whenever neutrinos goes across the boundary between two media with different indices of refraction. This effect occurs in the context of the standard model and does not depend on any exotic neutrino property. We examine such a phenomena and compare it with the transition radiation of a neutrino endowed with an intrinsic dipole moment.
\end{abstract}

The electromagnetic properties of neutrinos are of great relevance in a variety of physical, astrophysical, and cosmological contexts [1]. From the experimental side, up to now there is no evidence confirming a nonzero value for any of these properties [2]. Any observed deviation from the predictions of the standard model (SM), minimally extended to accommodate neutrino masses, would have a profound implication for the search of new physics $[3,4]$. The electromagnetic characteristic of the neutrinos can also serve to elucidate whether they are Dirac or Majorana fermions [5]. On the other hand, as is now well known, the basic properties of neutrinos that propagate through a medium can be substantially different compared to their properties in the vacuum. In particular, because of their weak interactions with the charged leptons and nucleons in a background, neutrinos acquire an effective coupling to the electromagnetic field $[6,7]$. This fact can give rise to several interesting physical processes: plasmon decay $[8,9]$, absorption of electromagnetic waves [10], radiative neutrino decay [11], and Cherenkov radiation by chiral neutrinos $[12,13]$.

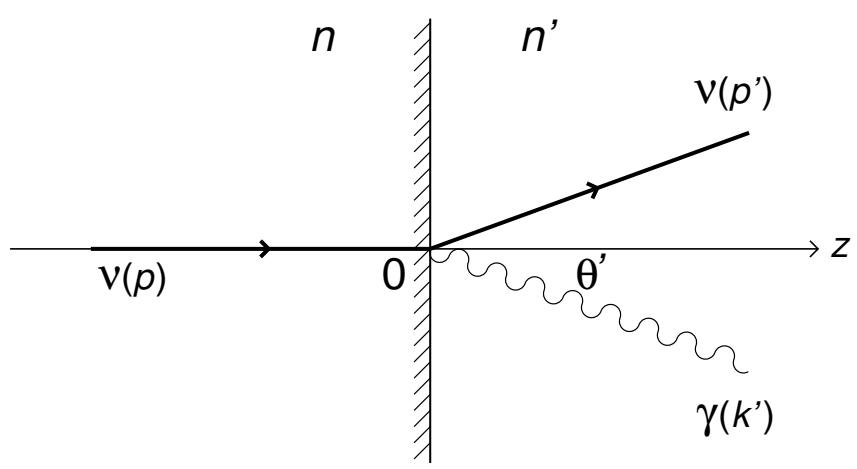

FIG. 1: Transition radiation at the interface between a medium with refraction index $n$ and the vacuum $\left(n^{\prime}=1\right)$.

In a uniform medium, the radiative process

$$
\nu(p) \rightarrow \nu\left(p^{\prime}\right)+\gamma(k)
$$

is kinematically allowed if $n>1$ and $v>1 / n$, where $n$ is the refraction index of the medium and $v$ the neutrino velocity. This leads to the Cherenkov radiation. There exists another important radiative process that can take place even if the above conditions are not satisfied: the transition radiation (TR) [14]. Such radiation is emitted whenever a charged particle goes across the boundary between two media with different indices of refraction. The phenomenon also happens with a neutral particle having a non vanishing dipole moment. The TR of a neutrino produced by an intrinsic (magnetic, electric, or toroidal) dipole moment have been examined by several authors [15-17] and an application of such effect as a new technique to measure the neutrino magnetic moment has been proposed by Sakuda [15].

As was pointed out several years ago [13], when neutrinos cross the interface of two media they emit TR because of their effective electromagnetic interaction in matter [18]. To our knowledge this process has not received the attention it deserves, despite the fact that is a novel physical prediction that does not hinge on hypothetical neutrino properties and/or interactions beyond those of the SM. Hence, it has to be there and needs to be properly characterized in order

\footnotetext{
*Electronic address: dolivo@nucleares.unam.mx

†Electronic address: aloza@ibt.unam.mx
} 
to distinguish it from a similar effect that could be associated with new physics. In this paper we present a quantum theoretical calculation of the TR due to the neutrino-photon coupling in an electron background. As we show, the effect under consideration can be comparable, and even larger, than the one due to an intrinsic electromagnetic characteristic of the neutrino, and therefore should be taken into account when analyzing a TR experiment eventually designed to measure the neutrino magnetic moment.

Let us consider a neutrino beam crossing the plane interface between a material medium and the vacuum, as shown in Fig. 1. For definiteness, we restrict ourselves to the case where neutrinos go from the medium into the vacuum, but a similar calculation can be done for the reverse situation or the case where instead of the vacuum we have another material medium. We choose the coordinate system in such a way that, in the frame where the medium is at rest, the interface coincides with the plane at $z=0$ and the medium occupy the region located at $z<0$. In this frame, $p^{\mu}=(\mathcal{E}, \wp)$ and $p^{\prime \mu}=\left(\mathcal{E}^{\prime}, \wp^{\prime}\right)$ are the components of the initial and final momenta of the neutrino. The incident neutrinos are assumed to move along the $z$ axis, perpendicularly to the interface, that is $\wp=\wp \hat{\mathbf{z}}$, with $\wp=|\wp|$. The four momentum of the emitted photon is $k^{\mu}=(\omega, \boldsymbol{\kappa})$ in the medium and $k^{\prime \mu}=\left(\omega^{\prime}, \boldsymbol{\kappa}^{\prime}\right)$ in the vacuum, with $\omega=\omega^{\prime}$ but $\boldsymbol{\kappa} \neq \boldsymbol{\kappa}^{\prime}$ due to the nonconservation of the momentum along the $z$ direction.

The influence of the medium on the energy momentum relation of the neutrinos does not play an essential role in our analysis and we take $p^{2}=p^{\prime 2}=m_{\nu}^{2}$, where $m_{\nu}$ is the neutrino mass. On the contrary, in general it is not correct to disregard the matter effects on the photon dispersion relation $\omega(\kappa)$. They can be determined from the background contributions to the polarization tensor $\pi_{\mu \nu}$. In an isotropic medium [19, 20]

$$
\pi_{\mu \nu}(k)=\pi_{T} R_{\mu \nu}+\pi_{L} Q_{\mu \nu}
$$

where

$$
\begin{aligned}
& R_{\mu \nu}=g_{\mu \nu}-\frac{k_{\mu} k_{\nu}}{k^{2}}-Q_{\mu \nu}, \\
& Q_{\mu \nu}=-\frac{k^{2}}{\kappa^{2}}\left(u_{\mu}-\frac{\omega}{k^{2}} k_{\mu}\right)\left(u_{\nu}-\frac{\omega}{k^{2}} k_{\nu}\right),
\end{aligned}
$$

are mutually orthogonal tensors $\left(R_{\mu \nu} Q^{\mu \nu}=0\right)$ that satisfy the relations $R_{\mu \nu} R^{\mu \nu}=2$ and $Q_{\mu \nu} Q^{\mu \nu}=1$. Here, $u^{\mu}=(1, \mathbf{0})$ is the four velocity of the medium, while the coefficients $\pi_{T, L}(\omega, \kappa)$ are scalar functions of the invariant quantities $\omega=k \cdot u$ and $\kappa=\sqrt{\omega^{2}-k^{2}}$, with $\kappa=|\kappa|$.

Since the longitudinal mode (plasmon) does not propagate in vacuum, only the two degenerated transverse modes (photon) contribute to the processes we are interested in. The photon frequency within the medium is given by the proper dispersion relation determined from the solution to

$$
\omega^{2}(\kappa)-\kappa^{2}=\pi_{T}(\omega(\kappa), \kappa)
$$

A common practice is to express $\omega(\kappa)$ in terms of the index of refraction $n \equiv \kappa / \omega(\kappa)$. The quantity of interest is the energy $\mathcal{S}$ radiated forward, into the vacuum. This is computed from the transition probability $\mathcal{W}$ for the process in Eq. (1)

$$
d \mathcal{W}=\frac{V d^{3} \wp^{\prime}}{(2 \pi)^{3}} \frac{V d^{3} \kappa^{\prime}}{(2 \pi)^{3}}\left|S_{f i}\right|^{2}
$$

where

$$
\begin{aligned}
& \left|S_{f i}\right|^{2}=\frac{\pi^{3}}{v V^{2}} \frac{|\mathcal{M}|^{2}}{\mathcal{E} \mathcal{E}^{\prime} \omega} \delta\left(\wp_{x}-\wp_{x}^{\prime}-\kappa_{x}\right) \delta\left(\wp_{y}-\wp_{y}^{\prime}-\kappa_{y}\right) \\
& \times \delta\left(\mathcal{E}-\mathcal{E}^{\prime}-\omega\right)\left|\int_{-\ell / 2}^{0} \mathrm{~d} z \exp \left[i\left(\wp-\wp_{z}^{\prime}-\kappa_{z}\right) z\right]\right|^{2} .
\end{aligned}
$$

Here, $V=\ell^{3}$ denotes the volume of the transition region and $v=\ell / \tau$ is the neutrino velocity expressed in terms of the time interval of the process $\tau$. In writing Eq. (7) we used the fact that, for the neutrino-photon interaction in matter, the matrix element $S_{f i}$ vanishes outside of the medium $(z>0)$.

The emitted photon is described by a monochromatic wave with a definite (transverse) polarization vector $\epsilon_{\mu}(k, \lambda)=$ $(0, \boldsymbol{\epsilon}(k, \lambda))(\lambda=1,2)$, which satisfies $\epsilon(k, \lambda) \cdot k=\epsilon(k, \lambda) \cdot u=0$ and $\epsilon(k, \lambda) \cdot \epsilon\left(k, \lambda^{\prime}\right)=\delta_{\lambda \lambda^{\prime}}$. Thus, for the $\nu \nu \gamma$ amplitude we have

$$
\mathcal{M}=-i \sqrt{\mathcal{N}} \bar{u}\left(p^{\prime}\right) \Gamma^{\mu} u(p) \epsilon_{\mu}(k, \lambda)
$$




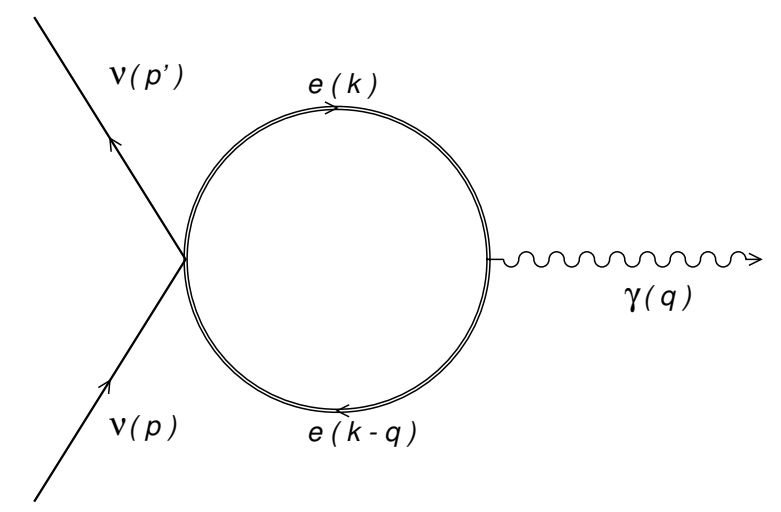

FIG. 2: One-loop diagram contributing, in the local approximation, to the neutrino-photon vertex in matter. The internal double lines represent the electron thermal propagators.

where $u(p)$ represents the Dirac spinor with momentum $p$. The factor $\sqrt{\mathcal{N}}$ has to be included because the normalization of the photon wave function in the medium differs from the one in vacuum [9]. The electromagnetic vertex function $\Gamma^{\mu}$ refers to the background part and depends not only on the momenta of the ingoing and outgoing neutrino, but also on $u^{\mu}$.

In many circumstances electrons make the dominant contribution to the neutrino electromagnetic vertex and, in a first approximation, we ignore the contributions coming from the protons and neutrons present in the background ${ }^{1}$. The general expression for $\Gamma^{\mu}$ in a medium consisting of an electron gas has been explicitly calculated to lowest order in the Fermi coupling constant $G_{F}$ [6]. In the local approximation, i.e, neglecting the momentum dependence in the weak bosons propagators the contributions to the $\nu \nu \gamma$ vertex reduce to the single diagramm shown in Fig. 2. From the result presented in the works of Ref. [6] we can write

$$
\Gamma_{\mu}=-\sqrt{2} \frac{G_{F}}{e}\left(a \pi_{\mu \nu}+b \pi_{\mu \nu}^{A}\right) \gamma^{\nu} L
$$

where $L=\frac{1}{2}\left(1-\gamma_{5}\right)$ and $e$ is the electric charge of the electron. In the $\operatorname{SM} a=2 \sin ^{2} \theta_{W} \pm \frac{1}{2}$ and $b=\mp \frac{1}{2}$, where the upper sign corresponds to the $\nu_{e}$ and the lower sign to the $\nu_{\mu, \tau}$. The first and second term in Eq. (9) correspond to the contribution coming from the vector and axial vector part of the electron current, respectively, in the effective four-fermion interaction between electrons and neutrinos.

The formula for $\pi_{\mu \nu}$ is the one given in Eq. (2), while $\pi_{\mu \nu}^{A}=\pi_{A}(\omega, \kappa) P_{\mu \nu}$, with $P_{\mu \nu}=i \epsilon_{\mu \nu \alpha \beta} k^{\alpha} u^{\beta} / \kappa$. It can be immediately verified that $k^{\mu} \pi_{\mu \nu}=k^{\mu} \pi_{\mu \nu}^{5}=0$, which in turn implies that $k^{\mu} \Gamma_{\mu}=0$ as required by gauge invariance. At this point, it is pertinent to note that the most general expression for $\pi_{\mu \nu}$ includes a term proportional to the tensor $P_{\mu \nu}$. For a parity-conserving media such a term can arise only through higher-order contributions to the photon self-energy involving the weak interaction of the particles present in the background and hence must be small [20]. Consequently, we ignored it in writing Eq. (2), thus taking the transverse modes degenerated.

Performing the integrations on $d^{3} \wp^{\prime}$ the energy radiated into the vacuum becomes

$$
\frac{d^{2} \mathcal{S}}{d \omega d \theta^{\prime}}=\omega \frac{d^{2} \mathcal{W}}{d \omega d \theta^{\prime}}=\frac{1}{32 \pi^{2} v} \frac{\omega^{2}|\overline{\mathcal{M}}|^{2} \sin \theta^{\prime}}{\mathcal{E}_{\wp_{z}^{\prime}}^{\prime}\left(\kappa_{z}+\wp_{z}^{\prime}-\wp\right)^{2}},
$$

with $\kappa_{z}=\kappa \cos \theta$ and $\wp_{z}^{\prime}=\sqrt{\wp^{2}-2 \mathcal{E} \omega+\omega^{2} \cos ^{2} \theta^{\prime}}$. Here, $\theta$ and $\theta^{\prime}$ are the angles that the photon momentum makes with the $z$ axis within and outside the medium, respectively. They are related by the Snell's law $\sin \theta^{\prime}=n \sin \theta$ $\left(n^{\prime}=1\right)$, as a result of conservation of the transverse photon momentum $\left(\kappa_{x, y}=\kappa_{x, y}^{\prime}\right)$. For $n$ close to 1, the distinction between $\theta$ and $\theta^{\prime}$ is not numerically significant, but it has to be kept to avoid a spurious singularity in the angular integration From a physical point of view, this is related to the fact that photons are emitted into the vacuum at angles smaller than $\pi / 2$.

\footnotetext{
1 The nucleon contributions to the neutrino electromagnetic vertex in matter, including the effect of the anomalous moment of the nucleons, has been calculated in Ref. [7]. They should be incorporated in a more general treatment of the problem.
} 
The quantity $|\overline{\mathcal{M}}|^{2}$ is calculated by averaging over the initial neutrino spins and summing over the neutrino final spins and the two photon polarizations. The transverse and axial polarization functions $\pi_{T}$ and $\pi_{A}$ are evaluated at the photon dispersion relation in the medium ${ }^{2}$. They are given by integrals over the momenta of the electrons and positrons in the background. The most comprehensive analytical expressions for these functions are those given by Braaten and Segel [21]. From them, one can show that in the classical, degenerate, and relativistic limit $\pi_{A} / \pi_{T} \lesssim$ $\sqrt{\alpha / 3 \pi}$, with $\alpha=e^{2} / 4 \pi$. As a result, we discard the contributions to $|\overline{\mathcal{M}}|^{2}$ that come from the axial vector part in $\Gamma_{\mu}$. This leads to

$$
|\overline{\mathcal{M}}|^{2} \cong \mathcal{N} \frac{G_{F}^{2}}{\pi \alpha} a^{2}\left|\pi_{T}\right|^{2}\left(\mathcal{E} \mathcal{E}^{\prime}-\wp \wp_{z}^{\prime} \cos ^{2} \theta+\kappa \wp \cos \theta \sin ^{2} \theta\right)
$$

where we took into account that $Q^{\mu \nu} \epsilon_{\mu}(k, \lambda)=0$ and used the relation $\sum_{\lambda=1,2} \epsilon_{\mu}(k, \lambda) \epsilon_{\nu}(k, \lambda)=-R_{\mu \nu}$ valid for the polarization vectors in the medium.

The energy spectrum is obtained by substituting (11) into (10) and integrating over the angular variable. The upper value for the angle in vacuum depends on $\omega$ and it is simpler to integrate over the angle in the medium, for which $0 \leqslant \theta \leqslant \pi / 2$. Then, using $d \theta^{\prime} / d \theta=n \cos \theta / \sqrt{1-n^{2} \sin \theta}$, for a relativistic neutrino $(\mathcal{E} \cong \wp)$ we find

$$
\frac{d \mathcal{S}}{d \omega}=\frac{\mathcal{N}}{v} \frac{G_{F}^{2} a^{2}}{32 \pi^{3} \alpha} \kappa^{2}\left|\pi_{T}\right|^{2} \mathcal{F}(\omega)
$$

where

$$
\mathcal{F}(\omega)=\int_{0}^{1} \frac{\mathrm{d} \zeta \zeta}{\sqrt{1-n^{2}\left(1-\zeta^{2}\right)}}\left[\frac{\wp-\omega-\wp_{z}^{\prime} \zeta^{2}+\kappa \zeta\left(1-\zeta^{2}\right)}{\wp_{z}^{\prime}\left(\wp_{z}^{\prime}+\kappa \zeta-\wp\right)^{2}}\right]
$$

with $\zeta=\cos \theta$. In writing Eq. (13) we took into consideration Snell's law and that in vacuum $\kappa^{\prime}=\omega$.

The last integral can not be done in a closed form, however a good analytic result for $\mathcal{F}(\omega)$ is obtained by putting $f(\zeta)=\sqrt{1-n^{2}\left(1-\zeta^{2}\right)}$ equal to one in the integrand. In fact, $f(\zeta)$ differs significantly from unity only for $n \approx 1$, but in this case the factor between square brackets exhibits a sharp maxima at $\zeta \approx 1$. Since $f(1)=1$ we can replace $f(\zeta)$ by one within the integral. Proceeding in this way we arrive to

$$
\frac{d \mathcal{S}}{d \omega}=\frac{\mathcal{N}}{v} \frac{G_{F}^{2} a^{2}}{64 \pi^{3} \alpha}\left|\pi_{T}\right|^{2}\left[\left(1-\frac{\omega}{\wp}\right) \mathcal{I}(s)-\frac{\wp^{2}}{2 \kappa^{2}} \mathcal{L}(s)+\frac{\wp^{2}}{4 \kappa^{2}}\left(\frac{2 \kappa^{2}}{\wp^{2}}-1+\varrho\right) \mathcal{J}(s)\right]_{s_{0}}^{s_{1}},
$$

where $\varrho=\left[(\wp-\omega)^{2}-\kappa^{2}\right] / \wp^{2}, s_{1}=(\wp-\omega+\kappa) / \wp$, and $s_{0}=|\varrho|^{1 / 2}$. The functions $\mathcal{I}(s), \mathcal{J}(s)$, and $\mathcal{L}(s)$ are given by

$$
\begin{aligned}
& \mathcal{I}(s)=\frac{1-\varrho}{1-s}+\frac{\varrho}{s}+2 \varrho \ln (1-s)-2 \varrho \ln s \\
& \mathcal{J}(s)=\frac{(1-\varrho)^{2}}{1-s}-\frac{\varrho^{2}}{2 s^{2}}(4 s+1)+(3 \varrho+1)(1-\varrho) \ln (1-s)+(3 \varrho-2) \varrho \ln s, \\
& \mathcal{L}(s)=\frac{s}{4}(s+4)+\frac{\varrho^{2}}{4 s^{2}}(4 s+1)+(1-\varrho)^{2} \ln (1-s)+(1-\varrho) \varrho \ln s .
\end{aligned}
$$

It is important to remark that the formula (14) has been derived without any assumption about the background. It can be applied to compute the energy emitted by a relativistic neutrino when it crosses the interface between the vacuum and an electron gas of any type.

From the expression of $\mathcal{N}$ as a function of $\omega[21]$ one can show that it is always close to unit and in the numerical calculations we take $\mathcal{N}=1$. On the other hand, the momentum $\kappa$ has to be expressed in terms of the photon energy by solving Eq. (5). According to the results of Ref. [21] this entails to finding the solution of the transcendental equation

$$
\omega^{2}-\kappa^{2}=\omega_{p}^{2}\left[1+\frac{1}{2} G\left(v_{*}^{2} \kappa^{2} / \omega^{2}\right)\right]
$$

\footnotetext{
2 Momentum is conserved at the interaction vertex, even though it is not in the whole process since, when photons are radiated into the vacuum, part of the momentum of the initial neutrino is taken up by the medium.
} 
where $v_{*} \leq 1$ is a "typical" velocity of the electrons in the plasma and the function $G$ is defined by

$$
G(x)=\frac{3}{x}\left[1-\frac{2 x}{3}-\frac{(1-x)}{2 \sqrt{x}} \log \frac{1+\sqrt{x}}{1-\sqrt{x}}\right], \quad 0 \leq x \leq 1 .
$$

A convenient simplification can be introduced by means of an iterative procedure. First, we solve Eq. (16) by expanding function $G$ in a power series and keeping the lowest order term. This gives us $\kappa^{2} / \omega^{2}=\left(\omega^{2}-\omega_{p}^{2}\right) /\left(\omega^{2}+\frac{1}{5} v_{*}^{2} \omega_{p}^{2}\right)$ that corresponds to the first relativistic correction to the photon dispersion relation. Next, we make $\kappa^{2} / \omega^{2}$ equal to this approximate value in the argument of $G$ on the right-hand side of Eq. (16), which renders us $\kappa$ as a new explicit function of $\omega$ :

$$
\kappa^{2} \cong \omega^{2}-\omega_{p}^{2}\left[1+\frac{1}{2} G\left(v_{*}^{2} \frac{\omega^{2}-\omega_{p}^{2}}{\omega^{2}+\frac{v_{*}^{2} \omega_{p}^{2}}{5}}\right)\right]
$$

This function works remarkably well in the whole range of frequencies and we use it to perform the numerical integration, over the interval $\left(\omega_{p}, \wp\right)$, involved in the calculation of the total energy $\mathcal{S}$ radiated into the vacuum.

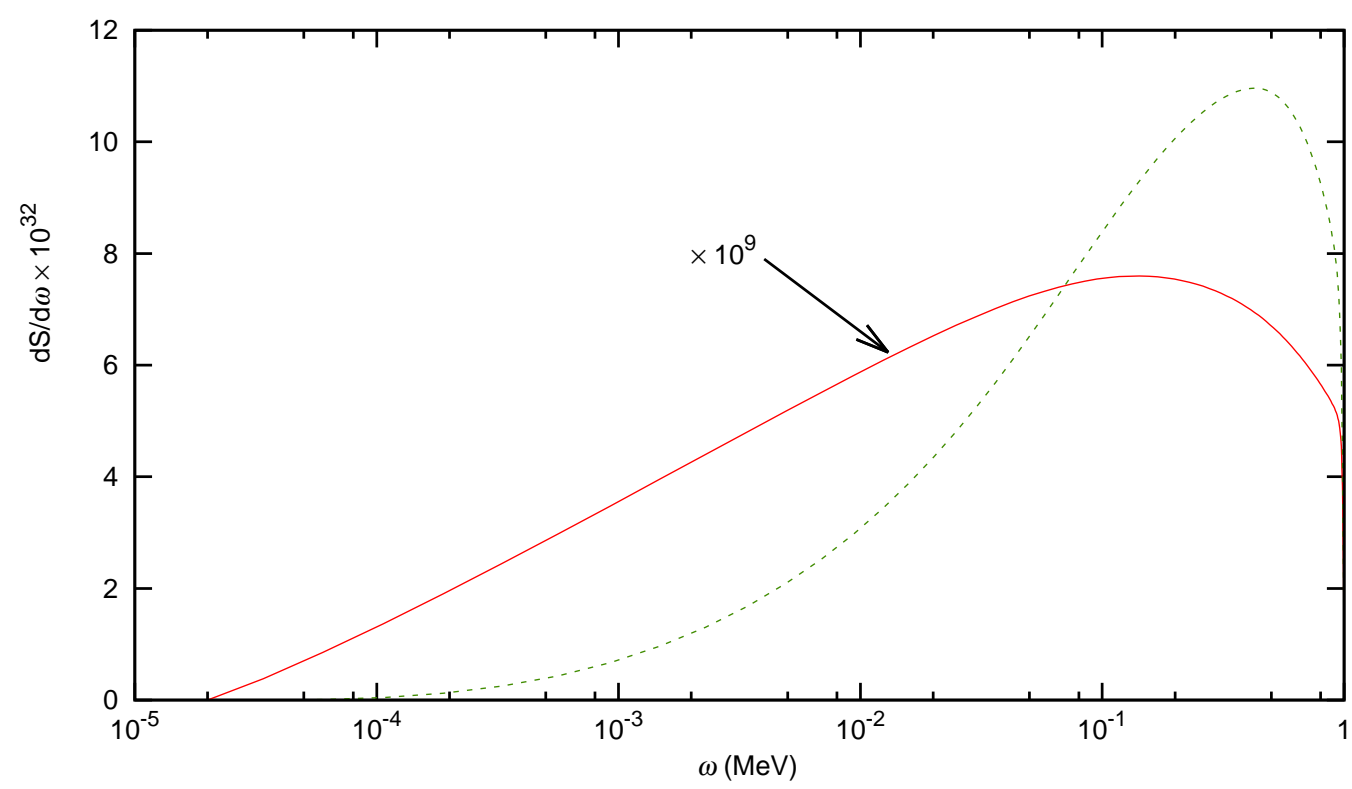

FIG. 3: Energy spectrum of the TR from the neutrino-photon coupling in matter for an incident neutrino with an energy $\mathcal{E}=1 \mathrm{MeV}$. The solid red and the dashed green lines correspond to a classical electron gas with $\omega_{p}=20 \mathrm{eV}$ and a degenerate gas with $\omega_{p}=5 \mathrm{KeV}$, respectively.

In Fig. 3 we have plotted the energy emitted by a $1 \mathrm{MeV}$ incident $\nu_{e}$ versus the photon energy, for two limiting situations: (i) a classical electron gas with $\omega_{p}=20 \mathrm{eV}$ (polypropylene) at room temperature $\left(v_{*}=\sqrt{5 T / m_{e}} \cong 0\right.$ ) and (ii) a degenerate gas $\left(v_{*}=v_{F}=0.3\right)$ with $\omega_{p}=5 \mathrm{KeV}^{3}$. As clearly illustrated in this figure, the intensity of the phenomenon and the shape of the spectrum depend markedly on the properties of the medium. For a degenerate plasma the TR energy is much higher and the spectrum is more sharp than in the classical limit. For the classical gas the total radiated energy in a single interface is $\mathcal{S} \cong 6.6 \times 10^{-35} \mathrm{eV}$, which is two orders of magnitude larger than the value obtained in the case of a toroidal dipole moment [17]. $[15]$

It is interesting to relate our result with the one due to a neutrino magnetic moment $\mu_{\nu}$. From Eq. (13b) of Ref.

$$
\mu_{\nu}=1.5 \times 10^{6} \mu_{B} \sqrt{\frac{\mathcal{S}_{M}}{\mathcal{E}}}
$$

\footnotetext{
${ }^{3}$ For a degenerate gas $\omega_{p}=(4 \alpha / 3 \pi) p_{F}^{2} v_{F}$ and $n_{e}=p_{F}^{3} / 3 \pi$, where $v_{F}=p_{F} / E_{F}$ and $n_{e}$ is the electron number density.
} 
where $\mu_{B}$ is the Bohr magneton and $\mathcal{S}_{M}$ denotes the total energy radiated because of $\mu_{\nu}$. When evaluated in $\mathcal{S}_{M}=$ $6.6 \times 10^{-35} \mathrm{eV}$ and $\mathcal{E}=1 \mathrm{MeV}$, the above formula yields $\mu_{\nu} \cong 1.2 \times 10^{-14} \mu_{B}$, which is several order of magnitude larger than the prediction of the minimally extended $\mathrm{SM}\left(\mu_{\nu} \approx 3 \times 10^{-19} \mu_{B}\left[m_{\nu} / 1 \mathrm{eV}\right]\right)$. The energy radiated by a magnetic moment increases, and becomes higher than the one predicted here, if we take a larger value for $\mu_{\nu}$, close to the present experimental limits. However, two things should kept in mind: i) such limits might be quite poor, as astrophysical arguments seem to indicate $[2,22]$, and ii) the process under consideration is a real one, in the sense that it represents a firm prediction based solely on the physics of the SM. Moreover, a $\mu_{\nu} \sim 10^{-14} \mu_{B}$ is of the same order as to the upper bound on the magnetic moments of Dirac neutrinos generated by physics above the scale of electroweak symmetry breaking [3].

A practical TR detector consists of several sets of a radiator, with a stack of thin foils of a certain material, to produce the TR photons and a gas proportional chamber to detect them. In the works of Refs. [15] and [17] the authors consider a detector of $10 \mathrm{~m}^{2}$ area made up of 10 sets of radiators and xenon chambers, each radiator comprising $10^{4}$ polypropylene foils. In our case, for the same experimental setup and a flux of $10^{13} \mathrm{~cm}^{-2} \mathrm{~s}^{-1}$ antineutrinos coming from a nuclear reactor, we get $W=2.1 \times 10^{-4}[\mathcal{T} / 1$ year $] \mathrm{eV}$, with $W$ being the total energy deposited during a time interval $\mathcal{T}$. Such a small value precludes its possible experimental observation. The measurement feasibility could be improved by enlarging the detector, for example, augmenting the number of foils. Finally, let us notice (see Eqs. (14) and (16)) that the radiated energy goes like $\omega_{p}^{4}$ and, as indicated before, increases enormously if, instead of a classical gas, we consider a degenerate electron plasma similar to those existing in stellar objects. The calculations presented in this paper are a first step towards a full understanding of this radiative neutrino phenomena and serve as a useful framework for further studies of its implications in such astrophysical environments and other physical situations.

\section{Acknowledgments}

The authors acknowledge support by DGAPA-UNAM under Grant No. PAPIIT IN117210 and by CONACyT under Grant No. 83534 and Red FAE.

[1] For a recent review see C. Giunti and A. Studenikin, Phys. Atom. Nucl. 32, 2089 (2009).

[2] G. Raffelt, Phys. Rep. 320, 319 (1999).

[3] N. F. Bell et al., Phys. Rev. Lett. 95, 151802 (2005); N. Bell, Int. J. Mod. Phys. A 22, 4891 (2007).

[4] H. Novales-Sánchez, A. Rosado, V. Santiago-Olán, and J. J. Toscano, Phys. Rev. D78, 073014 (2008).

[5] J. F. Nieves, Phys. Rev. D 26, 3152 (1982); B. Kayser, Phys. Rev. D 26, 1662 (1982); Phys. Rev. D 30,1023 (1984).

[6] V. N. Oraevskiŭ, V. B. Semikoz, and Ya. A. Smorodinskiı̆, JETP Lett. 43, 709 (1986); V. N. Oraevskiı̆, A. Yu. Plakhov, V. B. Semikoz, and Ya. A. Smorodinskiŭ, Sov. Phys. JETP 66, 890 (1987); Erratum ibid. 68, 1309 (1989); J. C. D’Olivo, J. F. Nieves, and P. B. Pal, Phys. Rev. D 40, 3679 (1989).

[7] J. C. D'Olivo and J. F. Nieves, Phys. Rev. D 56, 5898 (1997).

[8] J. B. Adams, M. A. Ruderman, and C. H. Woo, Phys. Rev. 129, 1383 (1963); G. Beaudet, V. Petrosianand E. E. Salpeter, Astrophys. J. 150, 979 (1967).

[9] M. H. Zaidi, Nuovo Cimento A 40, 502 (1965).

[10] V. N. Tsytovich, Sov. Phys. JETP 18, 816 (1964).

[11] J. C. D'Olivo, J. F. Nieves, and P. B. Pal, Phys. Rev. Lett. 64, 1088 (1990); D. Grasso and V. Semikoz, Phys. Rev. D 60, 053010 (1999).

[12] R. F. Sawyer, Phys. Rev. D 46, 1180 (1992).

[13] J. C. D'Olivo, J. F. Nieves, and P. B. Pal, Phys. Lett. B 365, 178 (1996).

[14] V. L. Ginzburg and I. Frank, J. Phys. (USSR) 9, 353 (1945); V. L. Ginzburg and V. N. Tsytovich, Transition Radiation and Transition Scattering, (Adam Hilger, New York, 1988).

[15] M. Sakuda, Phys. Rev. Lett. 72804 (1994); M. Sakuda and Y. Kurihara, Phys. Rev. Lett. 74,1284 (1995).

[16] W. Grimmus and H. Neufeld, Phys. Lett. B 344, 252 (1995).

[17] E. N. Bukina, V. M. Dubokiv, and V. E. Kuznetsov, Phys. Lett B 435, 134 (1998).

[18] J. C. D'Olivo and J. A. Loza, Transition Radiation of Neutrinos. Talk presented (JAL) at the VIII Latin American Symposium on High Energy Physics (SILAFAE 2010), Valparaíso, Chile, December 6-12, 2010. (Unpublished).

[19] H. A. Weldon, Phys. Rev. D 26, 1394 (1982).

[20] J. F. Nieves and P. B. Pal, Phys. Rev. D 39, 652 (1989); Erratum-ibid. D 40, 2148 (1989).

[21] E. Braaten and D. Segel, Phys. Rev. D 48, 1478 (1993).

[22] A. Ayala, J C. D'Olivo, and M. Torres, Phys. Rev. D59, 11190 (1999). 